\title{
The perception of size in a distorted room
}

WALTER C. GOGEL ${ }^{2}$ AND DONALD H. MERSHON UNIVERSITY OF CALIFORNIA, SANTA BARBARA

This study was concerned with determining the factors important in the size illusion occurring with respect to extraneous objects introduced into an Ames' monocular distorted room. It was asserted that the distortion in the room was unnecessary in order for the size illusion to be present. A rectangular room plus the extraneous objects produces the same stimulus conditions found in the Ames' room and, hence, should produce the same illusion in perceived size. In an experiment using a rectangular room, 24 Os judged the size and distance of objects at the same or different distances behind the windows. As in the case of the Ames' room it was found that the objects of physically equal size were perceived to be in the plane of the windows with the more distant objects appearing to have the smaller size. These results are explained in terms of the action of the equidistance tendency making the objects appear at the distance of the windows and the concept that objects in the same apparent fronto-parallel plane must have equal values of perceived size per unit of retinal size.

Objects of the same physical size placed in the windows of an Ames' distorted room appear to be of unequal size. Specifically, the object that appears in the physically more distant of the two apparently equidistant windows appears to be the smaller object. The usual explanation of the apparent difference in the sizes of the objects is that the familiarity that would indicate that the two objects are the same, or approximately the same, size is in conflict with the assumption that the room is rectangular (Ittelson \& Kilpatrick, 1961). It has seemed reasonable to suppose, since the size illusion usually is not encountered with rectangular rooms, that the erroneous perception of size is related to the distortion in the room. The present experiment demonstrates that this supposition is incorrect. It is shown that the same sort of error in perceived size can occur with a rectangular room and that the explanation of this error is found in quite a different phenomenon than that of the distortion of the room.

A schematic diagram illustrating the significant stimulus conditions of both an Ames' distorted room and the rectangular room used in the present study is shown in Fig. 1. In Fig. 1, the letters without primes represent physical positions in the Ames' distorted room, the letters with primes represent apparent positions in the Ames' distorted room and both physical and apparent positions in a rectangular room. The position of the observer $(0)$ is indicated at the front of the distorted room ABCD. Two windows, $a b$ and $\mathrm{cd}$, on the back wall of the distorted room are of equal angular size and appear at $a^{\prime} b^{\prime}$ and $c^{\prime} d^{\prime}$ along $B^{\prime} C^{\prime}$, the apparent orientation of the back wall of the room. Objects ef and gh are two objects of physically equal size located at different distances from $\mathrm{O}$. Since the angular size of ef is less than that of gh, and since the perceived distance of both of these is the same, it follows that the perceived size e' $f^{\prime}$ will be less than $g^{\prime} h^{\prime}$. The perception that $e^{\prime} f^{\prime}$ is less than $g^{\prime} h^{\prime}$, even though ef and gh are physically of equal size, is the size illusion associated with the distorted room. It has been found that the distortion in size between objects in the windows occurs between nonfamiliar (nonrepresentational) objects as well as between familiar objects (Epstein, 1962). Nonfamiliar objects were used in the windows in the present study.

In the description of the size illusion, it is clear that an identical stimulus configuration would have occurred if the wall $\mathrm{BC}$ had actually been located at $B^{\prime} C^{\prime}$ with the windows at $a^{\prime} b^{\prime}$ and $c^{\prime} d^{*}$, leaving the physical sizes and distance positions of ef and gh unchanged. In other words, a rectangular room with rectangular windows and with the two equally sized objects ef and gh presented at different distances behind the windows would result in the same stimulus events and, therefore, the same size illusion as in the case of the distorted room. Clearly, the significant question for the size illusion is why ef and gh appear at the distances of the apparently equally distant windows either with the distorted room or with the rectangular room. With either type of room a possible perception would have been that equally sized objects were located at different distances behind the windows. It is asserted that the reason why the two objects appeared at the distances of the apparently equidistant windows can be found in the action of the equidistance tendency. The equidistance tendency is a tendency for objects or parts of objects to appear equidistant with the strength of the tendency inversely related to directional separation (Gogel, 1965). As has been demonstrated, the equidistance tendency is more effective when cues to the physical distance between the objects are reduced or absent. Therefore, if there are few or no cues of relative depth between each object (ef or gh) and its directionally adjacent window, it would be predicted from the equidistance tendency that each object would appear at the depth of its window.

\section{Apparatus}

\section{METHOD}

A rectangular room, $34.3 \mathrm{~cm}$ high, $55.9 \mathrm{~cm}$ wide, and $62.2 \mathrm{~cm}$ deep, was viewed monocularly by $\mathrm{O}$ (with his right eye) through a $1 \mathrm{~mm}$ pinhole located $20.8 \mathrm{~cm}$ in front of the near wall of the room. The far wall of the room had a luminance of $60 \mathrm{ft}-\mathrm{L}$ and was formed by translucent material illuminated from behind by fluorescent light. The far wall contained two windows $(14 \mathrm{~cm}$ square) separated laterally by $7.3 \mathrm{~cm}$. Above each of the windows

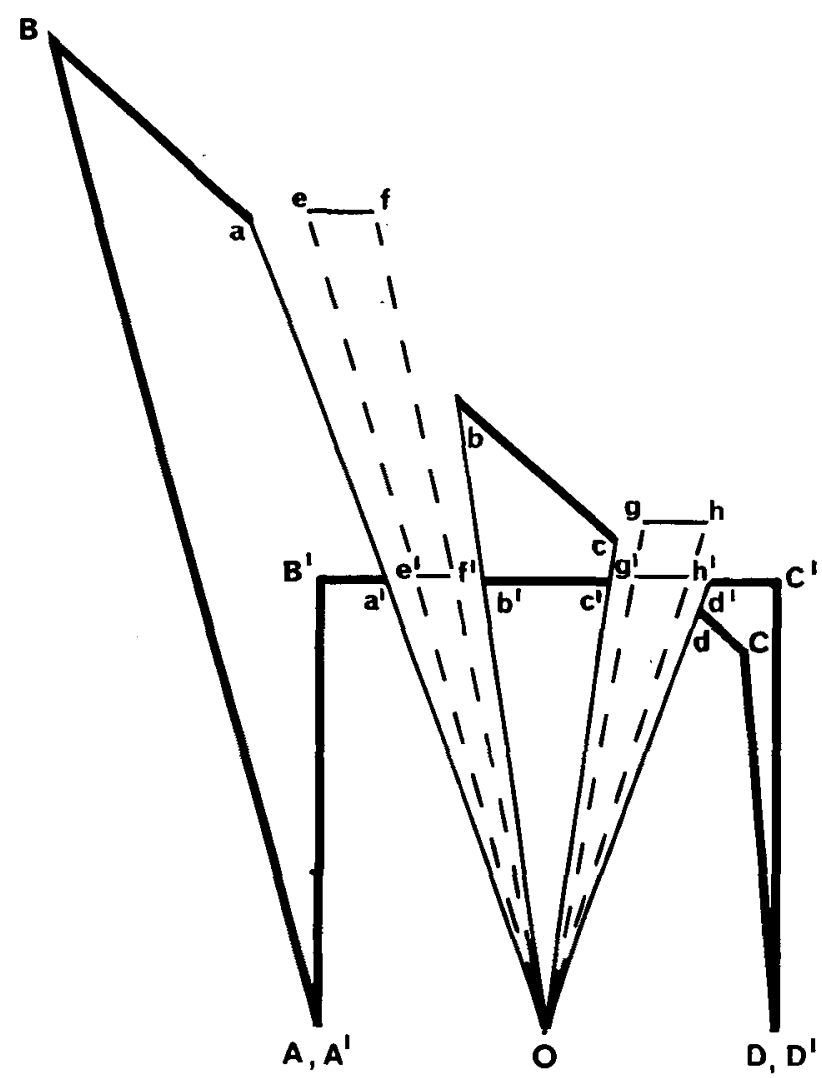

Fig. 1. A schematic top-view drawing of physical and apparent positions in an Ames' monocular distorted room and equivalent positions in a rectangular room. 
Table 1

Results from Size and Distance Estimates of the Lines Above the Windows. All values are given in $\mathrm{cm}$. The lines were $8 \mathrm{~cm}$ wide and both were located at the physical distance of $83.5 \mathrm{~cm}$ from 0 .

\begin{tabular}{lcccc} 
& $\begin{array}{c}\text { Verbal Reports } \\
\text { Perceived } \\
\text { Distance (D') }\end{array}$ & $\begin{array}{c}\text { Perceived } \\
\text { Size (S') }\end{array}$ & $\begin{array}{c}\text { Pointer } \\
\text { Adjustments } \\
\text { of size }\left(\mathrm{s}^{\prime}\right)\end{array}$ & $\begin{array}{c}\text { Reports of } \\
\text { depth (d') } \\
\text { between lines }\end{array}$ \\
\hline Mean & 343.2 & 44.0 & 8.4 & 3.8 \\
Median & 304.8 & 31.8 & 8.2 & 0.0 \\
SD & 282.5 & 39.2 & 1.2 & 24.1 \\
\hline
\end{tabular}

was a black, horizontal line $8.0 \mathrm{~cm}$ long. Behind each window was a hollow enclosure extending approximately $45.7 \mathrm{~cm}$ behind the window along lines of sight from $O$. These enclosures permitted stimuli (ef or gh of Fig. 1) to be presented either immediately behind the windows $(85.7 \mathrm{~cm}$ from O's eyes) or $42.5 \mathrm{~cm}$ behind the windows. The stimulus objects (ef or gh) were square tilted figures (diamonds) or circular figures (discs) cut out of opaque photographic film and mounted on cardboard carriers. The horizontal diagonal of the diamond and the diameter of the disc were both $6.0 \mathrm{~cm}$. Another white, homogeneous, transilluminated surface $(414 \mathrm{ft}-\mathrm{L})$ was visible through the cutout area of the stimulus figures. The floor of the room was composed of a checkerboard pattern of light and dark squares $(5.1 \mathrm{~cm}$ on a side). The walls and ceiling were light gray.

The apparatus was such that when $O$ looked into the room, he perceived a rectangular room with a checkerboard floor and with a white far wall on which were located two black squares (windows). In each window $O$ saw a luminous figure (diamond or disc), and above each window $O$ saw a short horizontal line. Also present

Table 2

Perceived Relative Depth d' and Perceived Relative Width s' in $\mathrm{cm}$ from the Objects Presented in Pairs.

The physical width of the objects was $6 \mathrm{~cm}$ and the physical depth between objects of a pair was either zero (Same Distances) or $40.3 \mathrm{~cm}$ (Different Distances).

Objects at Different Distances

\begin{tabular}{|c|c|c|c|c|c|c|c|}
\hline \multirow{2}{*}{$\begin{array}{l}\text { Objects } \\
\text { in pairs }\end{array}$} & \multirow[b]{2}{*}{ Position } & \multicolumn{3}{|c|}{$s^{\prime}$} & \multicolumn{3}{|c|}{$d^{\prime}$} \\
\hline & & Mean & Median & SD & Mean & Median & SD \\
\hline Diamond & Near & 6.1 & 5.8 & 1.4 & \multirow{3}{*}{7.4} & \multirow{3}{*}{0.0} & \multirow{3}{*}{34.9} \\
\hline Diamond & Far & 4.1 & 3.9 & 0.8 & & & \\
\hline Difference & & $2.0^{* *}$ & 1.9 & & & & \\
\hline Disc & Near & 6.5 & 6.4 & 1.1 & \multirow{3}{*}{5.7} & \multirow{3}{*}{0.0} & \multirow{3}{*}{22.5} \\
\hline Disc & Far & 4.3 & 4.2 & 0.8 & & & \\
\hline Difference & & $2.2^{* *}$ & 2.2 & & & & \\
\hline Diamond & Near & 6.1 & 5.9 & 1.1 & \multirow{3}{*}{7.3} & \multirow{3}{*}{0.0} & \multirow{3}{*}{20.8} \\
\hline Disc & Far & 4.4 & 4.4 & 0.8 & & & \\
\hline Difference & & $1.7^{* *}$ & 1.5 & & & & \\
\hline Disc & Near & 6.8 & 6.7 & 1.0 & \multirow{3}{*}{2.6} & \multirow{3}{*}{0.0} & \multirow{3}{*}{24.2} \\
\hline Diamond & Far & 4.2 & 4.2 & 0.7 & & & \\
\hline Difference & & $2.6^{* *}$ & 2.5 & & & & \\
\hline
\end{tabular}

\begin{tabular}{lllllllll}
\multicolumn{7}{c}{ Objects at Same Distances } \\
Objects & & \multicolumn{3}{c}{$\mathrm{s}^{\prime}$} & & & $\mathrm{d}^{\prime}$ & \\
in pairs & Position & Mean & Median & SD & & Mean & Median & SD \\
\hline Disc & Near & 6.8 & 6.6 & 1.1 & & & \\
Dismond & Near & 6.2 & 6.2 & 1.2 & 2.2 & 0.0 & 16.9 \\
Difference & & $0.6^{*}$ & 0.4 & & & & \\
Disc & Far & 4.4 & 4.4 & 0.8 & & & \\
Diamond & Far & 4.2 & 4.0 & 0.9 & 2.9 & 0.0 & 15.3 \\
Difference & & $0.2^{* *}$ & 0.4 & & & &
\end{tabular}

$p<.01$ ( $t$ wo-tailed $t$ test, $d f=23$ )

$* 0<.001$ (two-tailed t test, $d f=23$ ) at the base of the far wall were two vertical pointers, visible to 0 the lateral separation of which $O$ could adjust by turning a handle at the observation position. The adjusted separation of the two pointers was read by the experimenter (E) from a scale outside the room and invisible to $O$.

The observation position contained an adjustable chin rest, in addition to the pinhole and the handle for moving the pointers, and was located in a booth which could be made totally dark. A communication system was available. A shutter controlled by $\mathrm{E}$ occluded O's view when required.

\section{Subjects}

Twenty-four students from a summer session course in introductory psychology served as Os for this experiment. They were between the ages of 16 and 25 and all had a visual acuity of at least 20/20 in their right eyes (corrected with contact lenses, if necessary) as measured by a Keystone Orthoscope.

\section{Procedure}

At the start of the experiment, $O$ verbally indicated the distance from himself and the width (left-to-right extent) of the horizontal line located above one of the windows, with no luminous object present in either window. Following these judgments $O$ verbally indicated the distance, if any, that either of the two lines appeared to be behind the other. Following this, $O$ indicated the perceived width of the line above each of the windows by adjusting the distance between the black pointers at the base of the far wall until this distance appeared to be the same as the width of first one and then the other line. The pointers were reset by $E$ before each indication of size. For half the Os the pointers always started together; for the other half they started apart.

On following trials a pair of objects, either two diamonds, or two discs, or a diamond and a disc, were presented with one member of the pair directionally in the center of each window. The figures were either 2.2 (near position) or 42.5 (far position) $\mathrm{cm}$ behind the window. Each $\mathrm{O}$ was presented with a different balanced order of object pairs. With the following exceptions, all combinations were presented. (1) No $O$ was presented with two identical figures at the same time at the same distance. (2) For pairs of a diamond and a disc, half the Os were always presented with the diamond on the right and half with the diamond on the left. For each pair of objects, $O$ verbally indicated the depth, if any, which appeared between the objects and adjusted the black pointers to indicate the width of each object. The shutter at the observation position was closed. for $1 \mathrm{~min}$ between each presentation, during which time $\mathrm{E}$ changed the stimulus conditions as required.

The data from the experiment are summarized in Tables 1 and 2. The results in Table 1 labeled $D^{\prime}$ and $S^{\prime}$ are the average values (converted to centimeters) of the initial verbal reports of perceived distance from $O$, and of the perceived width, respectively, of the lines above the windows. The values of $D^{\prime}$ and $S^{\prime}$ serve the purpose of indicating the apparent distance to the far wall and the apparent size of objects at that distance. The distance to the far wall usually appeared much larger than its physical distance, indicating that extraneous distance cues were severely reduced in the experiment. As is shown by the standard deviation of the $D^{\prime}$ values, considerable variability occurred between $O s$ in this distance estimation. The ratio of $\mathrm{S}^{\prime} / \mathrm{D}^{\prime}$ computed from the average values of $S^{\prime}$ and $D^{\prime}$ was 0.128 , and the ratio of the physical width $S$ to the physical distance $D$ of the lines above the windows was 0.093 .

The results in Table 1 labeled $s^{\prime}$ and $d^{\prime}$ indicate the perceived width in $\mathrm{cm}$ of the lines, above the windows, as measured by the adjustment of the black pointers, and the perceived depth (converted to $\mathrm{cm}$ ) between the lines, respectively. As would be expected, the two lines appeared to be at approximately the same distance (with the left line, on the average, slightly behind the right) and to have approximately the same size. It will be noted that the average and median values of $S^{\prime}$ and $s^{\prime}$ in Table 1 differ 
markedly even though both are measures of the perceived width of the same lines. The reason for this difference is that $S^{\prime}$ is a "scalar" and $s^{\prime}$ a "nonscalar" measure of perceived extent (Gogel, 1968). A scalar measure of perceived extent indicates perceived extent in units that behaviorally have absolute values. For example, the $\mathbf{S}^{\prime}$ values are the values of width which $O$ might expect to find if he reached out to pick up the object. On the other hand, the units of the 's' measures have only relative behavioral values, indicating the perceived width of the lines relative to the lateral separation of the black pointers at the same apparent distance. The difference between these two types of measures of perceived size can be illustrated by considering what would occur if the visual field of $\mathrm{O}$ were suddently perceptually magnified by a factor of two. Under these circumstances the verbal report of perceived size $S^{\prime}$ would double, but the perceived size measured with the two black pointers s' would remain unchanged (Gogel, 1968). This inability of $s^{\prime}$ to reflect perceived absolute size, however, is of no consequence for this study since it is only required that changes in $s^{\prime}$ reflect changes in the relative perceived sizes of the several objects.

The results from the main part of the experiment are shown in Table 2. The values of $\mathrm{d}^{\prime}$ and $\mathrm{s}^{\prime}$ in Table 2 are the average measures of perceived depth $\left(d^{\prime}\right)$ between the objects in the windows and the average lateral adjustment of the black pointers ( $s^{\prime}$ ) a pparently equal to the width of each of the objects in the windows. For each pair of objects there are, of course, two values of $s^{\prime}$ but only one value of $\mathrm{d}^{\prime}$. The results indicate that, for each pair of objects, the perceived depth $d^{\prime}$ between the objects was close to zero. It seems that the equidistance tendency was quite effective in this study in making the objects in the windows appear very close to the windows in depth. The small values of $d^{\prime}$, although statistically nonsignificant at the .05 level, were in the direction of the physical depth between the objects when these were located at different distances. Probably, some slight cues to actual distance were present and were able to modify the tendency of the objects in the windows to appear at the distance of the windows. The values of $s^{\prime}$ in Table 2 associated with the diamonds and discs at different physical distances represent the main result of the experiment. They demonstrate the occurrence of the size illusion. Objects which were physically equal in width clearly appeared unequal, with the object subtending the smaller retinal size (the physically farther object) appearing smaller. The conditions in which the objects in the pair were at the same distance constitute a control indicating that diamonds and discs of the same retinal width, and at approximately the same apparent distance, have approximately the same apparent width. As can be seen from Table 2, the difference in apparent width between these figures, although significant, was very small compared with the main effects.

\section{DISCUSSION}

Although the present experiment is similar only to the Ames' demonstration in which objects appear behind the windows, it clearly applies in principle to the case in which objects appear of markedly different size within the distorted room. In the latter case also, objects of equal physical size but unequal angular size appear at the same distance and have different perceived sizes. In both cases, it is the equidistance tendency that is essential for the size illusion. The equidistance tendency, for example, tends to make the person appear at the intersection of the person's feet with the floor. This factor termed optical contact (Gibson, 1950, pp. 177-180), is a special case of the equidistance tendency (Gogel, 1965). It is clear that, if the retinal size of an object appearing in one corner of the far wall of a rectangular room is larger than the retinal size of an object appearing in the other (apparently equidistant) corner, the two objects will appear unequal in size.

The process involved in producing the size illusion in the prsesent experiment has two parts. The first part supported by the results from the present study, enphasizes the action of the equidistance tendency in making the equally sized objects at different physical distances appear at the same distance, i.e., at the distance of the apparently equidistant windows. The second part of the process (to be considered in later studies) concerns the factors determining the perceived size of each of the stimuli at this apparent distance. It might be supposed that the pesceived sizes of the objects are determined by their retinal sizes (cf. Rock \& McDermott, 1964) or that the objects are perceived relative to the windows (Rock \& Ebenholtz, 1959; Brown, 1965; Kunnapas, 1955). This latter alternative, however, is not in agreement with the results obtained by Epstein (1962). An additional possibility is that the perceived sizes of ef and gh of Fig. 1 can be explained in terms of their $S^{\prime} / \theta$ values, where $\Theta$ is the angular size of the width of an object whose perceived width is $S^{\prime}$. It is hypothesized (Gogel, 1964) that the difference in $S^{\prime} / \Theta$ values of two objects is a monotonic function of the difference in their perceived distances (and vice versa) with the apparently more distant object having the greater value of $S^{\prime} / \Theta$. Of particular interest for the present experiment is the corollary that objects at the same distance have equal values of $\mathbf{S}^{\prime} / \theta$. More generally, this corollary might be entitled the "context theory of perceived size." It follows that all parts of the far wall of the room, since they are apparently equidistant, have the same values of $S^{\prime} / \Theta$. Therefore, since the discs and diamond-shaped figures appear essentially at the distance of this wall, they must also have this value of $S^{\prime} / \Theta$. But, in the cases in which one of the objects is at a different physical distance from the other, the $\Theta$ values of the objects differ and, in order to have the same values of $S^{\prime} / \Theta$, it is necessary that they have different values of perceived size $S^{\prime}$.

It is clear from this study that a distorted room is not necessary to the perception of the size illusion with respect to the objects in the windows. According to the equidistance tendency and the context theory of perceived size, the basic processes involved in the size illusion associated with objects introduced into the Ames' distorted room does not require either that a distorted room be used or that the objects introduced be familiar objects or, indeed, even similar to each other in shape.

\section{REFERENCES}

BROWN, J. F. The visual perception of velocity. In J. M. Spiegel (Ed.), Readings in the study of visually perceived movement. New York: Harper \& Row, 1965. Pp. 64-107.

EPSTEIN, W. A test of two interpretations of the apparent size effects in a distorted room. J, exp. Psychol., 1962, 63, 124-128.

GIBSON, J. J. The perception of the visual world. New York: Houghton Mifflin, 1950.

GOGEL, W. C. Size cue to visually perceived distance. Psychol. Bull., 1964, $62,217-235$.

GOGEL, W. C. Equidistance tendency and its consequences. Psychol Bull, $1965,64,153-163$.

GOGEL, W. C. The measurement of perceived size and distance. In W. D. Neff (Ed.), Contributions to sensory physiology, Vol. III. New York: Academic Press, in press.

ITTELSON, W. H., \& KILPATRICK, F. P. The monocular and binocular distorted rooms. In F. P. Kilpatrick (Ed.), Explorations in transactional psychology. New York: New York University, 1961. Pp. 154-173.

KILPATRICK, F. P., \& ITTELSON, W. H. The size-distance invariance hypothesis. Psychol. Rev., 1953, 60, 223-231.

KUNNAPAS, T. M. Influence of frame size on apparent length af a line. $J$. exp. Psychol., 1955, 50, 168-170.

ROCK, I., \& EBENHOLTZ, S. The relational determination of perceived size. Psychol. Rev., 1959, 66, 387-401.

ROCK, 1., \& McDERMOTT, w. The perception of visual angle. Acta Psychol, 1964, 22, 119-134.

\section{NOTES}

1. This research was supported by Grant NGR-05-010-010 from the National Aeronautics and Space Administration.

2. Address: Department of Psychology, University of California, Santa Barbara, Santa Barbara, Calif. 93106.

(Accepted for publication February 26, 1968.) 